University of Nebraska - Lincoln

DigitalCommons@University of Nebraska - Lincoln

Faculty Publications, Department of Psychology

Psychology, Department of

September 2004

\title{
Nicotine serves as a feature-positive modulator of Pavlovian appetitive conditioning in rats
}

M. I. Palmatier

University of Nebraska-Lincoln

J. L. Peterson

University of Nebraska-Lincoln

J. L. Wilkinson

University of Nebraska-Lincoln

Rick A. Bevins

University of Nebraska-Lincoln, rbevins1@unl.edu

Follow this and additional works at: https://digitalcommons.unl.edu/psychfacpub

Part of the Psychiatry and Psychology Commons

Palmatier, M. I.; Peterson, J. L.; Wilkinson, J. L.; and Bevins, Rick A., "Nicotine serves as a feature-positive modulator of Pavlovian appetitive conditioning in rats" (2004). Faculty Publications, Department of Psychology. 39.

https://digitalcommons.unl.edu/psychfacpub/39

This Article is brought to you for free and open access by the Psychology, Department of at DigitalCommons@University of Nebraska - Lincoln. It has been accepted for inclusion in Faculty Publications, Department of Psychology by an authorized administrator of DigitalCommons@University of Nebraska - Lincoln. 
Palmatier MI, Peterson JL, Wilkinson JL, \& Bevins RA (2004) Nicotine serves as a feature-positive modulator of Pavlovian appetitive conditioning in rats. From Behavioural Pharmacology, 15, 183-194. Published by Lippincott Williams \& Wilkins; copyright (C) 2004 Lippincott Williams \& Wilkins. Used by permission. 


\title{
Nicotine serves as a feature-positive modulator of Pavlovian appetitive conditioning in rats
}

\author{
M. I. Palmatier, J. L. Peterson, J. L. Wilkinson and R. A. Bevins
}

The present experiments examined whether a nicotine state could set the occasion for a pairing between visual cues and a rewarding outcome in rats. Following nicotine administration, presentation of a conditional stimulus (CS; light-on) was followed by brief access to a sucrose solution. When saline was administered, the same CS was presented but was not followed by any consequence. In Experiment 1, two groups assessed whether rats could acquire this Pavlovian feature-positive discrimination via different training procedures. An anticipatory food-seeking conditioned response (CR) developed during the CS on nicotine sessions but not on saline sessions in both groups. In Experiment 2, centrally acting antagonists of nicotinic acetylcholine and opiate receptors (mecamylamine and naloxone, respectively) dose-dependently blocked nicotine's control of the CR, whereas the peripherally acting nicotinic antagonist hexamethonium had no effect. Increasing or decreasing the interval between nicotine administration and testing also attenuated the CR. These results are consistent with

\section{Introduction}

Drug discrimination is a widely used animal model for the self-reported or subjective effects of drugs in humans (Schuster and Johanson, 1988). One reason for the widespread use of this model is that behavior controlled by drug states is sensitive to pharmacological manipulations and reflects drug action at a cellular level (Holtzman and Locke, 1988), Traditionally, operant conditioning techniques are used to investigate the stimulus effects of drugs. In such tasks, the presence versus absence of an interoceptive drug stimulus guides schedule-maintained behavior. For example, following drug administration, a fixed number of responses on a lever (e.g. left) may be reinforced by food. Following saline administration, a distinctly different lever (e.g. right) is reinforced on the same schedule. As a result of this training, the drug cue becomes a discriminative stimulus $\left(S^{D}\right)$ that guides behavioral output. Thus, the stimulus effects of the drug set the occasion upon which left-lever pressing will be reinforced. Conversely, the absence of the drug effects sets the occasion on which right-lever pressing will be reinforced.

The investigation of drug discriminative stimuli has also been extended to aversive Pavlovian conditioning situations. This preparation, termed discriminated taste aversion, typically involves presenting a flavor conditional

0955-8810 (C) 2004 Lippincott Williams \& Wilkins the hypothesis that nicotine can occasion appetitive Pavlovian relations via its action at central nervous system cholinergic receptors. Behavioural Pharmacology 15:183-194 (c) 2004 Lippincott Williams \& Wilkins.

Behavioural Pharmacology 2004, 15:183-194

Keywords: drug discrimination, nicotine, opioid, mecamylamine, naloxone, appetitive conditioning, rat

Department of Psychology, University of Nebraska-Lincoln, Nebraska, USA.

Sponsorship: This research was partially supported by grants from NIH (DA11893) and the UN-L Research Council, and partially fulfills the requirements for the doctoral degree of the first author, who was supported by NIH grant DA16179A.

Correspondence and requests for reprints to Rick A. Bevins, Department of Psychology, University of Nebraska-Lincoln, Lincoln, NE USA 68588-0308. e-mail: rbevins1@unl.edu

Received 5 January 2004 Accepted as revised 29 April 2004

stimulus (CS) (e.g. saccharin solution) to water-deprived rats. When the flavor is presented in a drug state (e.g. morphine; Martin et al., 1990), it is followed by an aversive outcome (i.e. lithium chloride injection). In the absence of drug, access to the flavor is not followed by any consequence. By the end of training, rats drink more saccharin when morphine is absent than when morphine is present.

The discriminated taste aversion procedure is a useful tool for characterizing the behavioral and stimulus effects of drugs. However, due to the aversive nature of the associations (taste-illness), interpretation and extrapolation are necessarily confined to avoidance learning situations. One question that has not been answered sufficiently is whether the pharmacological effects of drugs can guide Pavlovian conditioned approach behavior. To our knowledge, two studies (Parker et al., 1994; Miller et al., 2002) represent the only attempts to use a drug state to control appetitive Pavlovian conditioning. In these studies, the pharmacological effects of a drug (i.e. methadone) resolved the ambiguity of two keylight stimuli. For example, the presence of methadone signaled that illumination of a green keylight was followed by access to grain. The absence of methadone signaled that grain followed illumination of a white keylight. Even though responding was not required to receive grain 
access, pigeons pecked more at the keylight that was followed by grain access in each drug state.

The fact that only two published studies (Parker $e t$ al., 1994; Miller et al., 2002) have examined the cueing properties of drugs in Pavlovian approach situations is surprising. Many modern theories of drug abuse implicate Pavlovian conditioning processes in maintenance and relapse of chronic drug use (e.g. Rose and Levin, 1991; Robinson and Berridge, 1993; Siegel et at., 2000; Koob and LeMoal, 2001). Notably, many of these models conceptualize drugs as outcomes (i.e. behavioral reinforcers or unconditional stimuli) paired with purportedly neutral environmental stimuli (but see Siegel et al., 2000). These 'neutral' environmental stimuli acquire the ability to evoke approach behavior as a result of this pairing. However, contemporary researchers are beginning to examine the interaction between appetitive stimuli and drugs of abuse. For example, Caggiula and colleagues (Donny et al., 2003) have demonstrated that noncontingent nicotine infusions enhance lever pressing for a reinforcing visual stimulus. Other laboratories have demonstrated that nicotine and amphetamine can enhance the approach or seeking behaviors controlled by environmental cues (e.g. tone/light or clicker) that are conditional signals for rewarding events (e.g. sucrose pellets or water access, see Wyvell and Berridge, 2000, 2001; Olausson et al., 2003; 2004a, b). Finally, some researchers have demonstrated that drug outcomes can be associated with other drug cues that differ in intensity and/or pharmacology (e.g. Revusky $e t$ al., 1989; Kim et al., 1999).

Recent work from our laboratory reflects a similar broadening of the role of Pavlovian conditioning processes in drug abuse. For example, we recognize that not all environmental cues are 'neutral'. In fact, some cues that reliably occur with the pharmacological effects of drugs will have positive motivational value. If the pharmacological effects of a drug were consistently paired with such stimuli, then the drug might acquire the ability to evoke approach or investigatory responses (see Alessi et al., 2002). Indeed, we have demonstrated recently that nicotine serves as a conditional stimulus for access to sucrose (Besheer et al., 2004). Briefly, pairing nicotine with a rewarding outcome (sucrose access) appeared to give nicotine some conditioned excitatory value as expressed by anticipatory food-seeking (i.e. goal tracking; Farwell and Ayres, 1979).

The experiments reported here extend our previous work by examining whether nicotine can control condirioned approach evoked by an environmental cue (i.e. light CS). In the nicotine state, this CS was immediately followed by sucrose access; no programmed outcome occurred when the CS was presented in a saline state. In this situation, we conceptualized nicotine as a 'positive feature' rather than a Pavlovian conditional stimulus (e.g. Bouton and Swartzentruber, 1986; Holland, 1999; Rescorla, 1986). That is, nicotine served as a cue that sers the occasion on which the light CS will be followed by sucrose. Throughout the remainder of this manuscript we refer to the nicotine cue as a 'feature' of the discrimination or a 'modulator' of conditional responding, in order to avoid terminologies that might infer behavioral mechanisms (i.e. conditioned facilitator; Rescorla, 1986; or Pavlovian occasion setter; Holland, 1983).

Experiment 1 established that goal tracking developed during the light CS, but only in the nicotine state. Followup tests explored some associative properties of nicotine in this situation. For example, we examined whether repeatedly presenting nicotine alone (i.e. extinction) affected goal tracking when nicotine and the light CS were subsequently tested together. In a similar preparation, we found that when nicotine was trained as a conditional stimulus for sucrose (i.e. no light cues), extinction of nicotine attenuated nicotine-evoked goal tracking (Besheer et al., 2004). Experiment 2 examined some of the pharmacological properties of the putative nicotine modulator. For example, both nicotinic acetylcholine and opiate receptor systems are involved in cigarette smoking (e.g. Brauer et al., 1999) and nicotine withdrawal (e.g. Malin et al., 1993, 1998). Therefore, we examined the effects of nicotinic acetylcholine receptor $(n A C h R)$ and opiate receptor antagonist pre-treatments on the ability of nicotine to occasion goal tracking to the light CS. Thus, Experiment 1 explored the behavioral conditions under which nicotine-specific goal tracking could be established and expressed, while Experiment 2 began to examine the pharmacological properties of nicotine in the Pavlovian feature-positive paradigm.

\section{Methods}

\section{Subjects}

Eighteen experimentally naïve male Sprague-Dawley rats were obtained from Harlan (Indianapolis, Indiana, USA) and housed individually in clear plastic tubs lined with wood-shavings in a temperature- and humiditycontrolled colony. Water was freely available; however, access to food was restricted, such that the rats were maintained at $85 \%$ of their free-feeding weight (mean $=320 \pm 7 \mathrm{~g}$ ). This target $85 \%$ body weight was increased by $2 \mathrm{~g}$ every 28 days to follow a typical growth curve provided by the supplier. Experimental sessions were conducted on consecutive days during the light portion of a $12 \mathrm{~h}$ light:dark cycle.

\section{Apparatus}

Seven standard operant chambers (Med Associates, Georgia, Vermont, USA) were housed in a polyvinyl chloride sound-attenuating cubicle, fitted with a fan to provide airflow and masking noise. Each chamber 
Table 1 Design of Experiment 1

\begin{tabular}{lcccc}
\hline Group & Training I (16 sessions) & Training II (40 sessions) & Extinction (6 sessions) & Tests \\
\hline Pre-training $(n=6)$ & Nic:L+ & Nic:L+/Sal:L- & Nic: - & Nic:L-/Sal:L- \\
Discrimination $(n=5)$ & Nic:L+/Sal:L- & Nic:L+/Sal:L- & Nic: - & Nic:L-/Sal:L - \\
\hline
\end{tabular}

Schematic of the procedures used in Experiment 1. Nic: or Sal: indicates that nicotine administration $10.4 \mathrm{mg} / \mathrm{kg} \mathrm{base,} \mathrm{s.c.)} \mathrm{or} \mathrm{saline} \mathrm{administration}(0.9 \%$, s.c.), respectively, preceded the session. $L$ indicates a 30 s presentation of light conditional stimulus (CS). + or - indicates access to $26 \%$ sucrose or emply interval immediately after each CS, respectively. / indicates separate sessions, which occurred on consecutive days in randomized order for each subject.

measured $30.5 \times 24.1 \times 21 \mathrm{~cm}(1 \times w \times h)$; the side walls were aluminum and all stimulus elements were attached to these walls. The ceiling and remaining walls of the chamber were constructed of clear polycarbonate. One side of each chamber was fitted with a liquid dipper in a receptacle measuring $5.2 \times 5.2 \times 3.8 \mathrm{~cm}(1 \times \mathrm{w} \times \mathrm{d})$. The dipper arm had a $0.1 \mathrm{ml}$ cup attached for sucrose delivery. The receptacle was fitted with an infrared emitter/ detector unit, $1.2 \mathrm{~cm}$ within the tray and $3 \mathrm{~cm}$ from the floor, to monitor head entries into the dipper. Two white stimulus lights $(100 \mathrm{~mA}, 2.54 \mathrm{~cm}$ diameter $)$ were mounted on the same wall, $14.6 \mathrm{~cm}$ above the floor and $3.5 \mathrm{~cm}$ from the nearest perpendicular wall. Illumination of these lights served as the CS. The apparatus was not fitted with a house light; however, some ambient light from the experimental room reached the closed cubicle through the fan and ventilation outlets. A personal computer with Med Associates interface and software controlled the stimulus events and recorded dipper entries throughout each session.

\section{Drugs}

(-)-Nicotine hydrogen tartrate, hexamethonium sulfate, mecamylamine hydrochloride, and naloxone hydrochloride (Sigma/RBI, St. Louis, Missouri, USA) were dissolved in saline and injected subcutaneously (SC) at a volume of $1 \mathrm{ml} / \mathrm{kg}$. Nicotine was brought to a $\mathrm{pH}$ of $7.0 \pm 0.2$ with a dilute $\mathrm{NaOH}$ solution. Nicotine doses are expressed as the base form. Doses of all other compounds are expressed as the salt form.

\section{Data analyses}

The number of dipper entries was recorded in 30-second (Experiment 1) or 15-second (Experiment 2) intervals. Dipper entries (i.e. goal tracking) were also recorded during the $4 \mathrm{~s}$ sucrose access periods. The main dependent measure was the average elevation score for each session. An elevation score for each trial was calculated from dipper entries that occurred during the light CS minus dipper entries that occurred during an equivalent pre-CS period (Brooks and Bouton, 1994). Thus, an average elevation score of 0 indicates equal goal tracking during the CS and pre-CS periods across a session; positive elevation scores indicate more goal tracking during CS presentations. Average elevation scores were analyzed with one-way or two-way analyses of variance (ANOVAs) where appropriate. For pharmacological antagonist and temporal generalization tests (Experiment 2), a significant one-way ANOVA was followed-up by Dunnett's multiple comparisons test, contrasting elevation scores from each condition with scores from the control condition. When follow-ups revealed significant differences, the test conditions were compared to a 'no-nicotine' control condition (using Bonferroni's error correction) that was derived from elevation scores on the first trial of two saline training sessions (see later). Statistical significance was set at $P \leq 0.05$ (two-tailed).

\section{Procedures: Experiment 1}

The protocol for each group is presented in Table 1 . Following dipper training (see later), rats were assigned randomly to one of two groups [Pre-training $(n=6)$ or Discrimination $(n=5)]$. Both groups received featurepositive discrimination training with nicotine $(0.4 \mathrm{mg} / \mathrm{kg})$ as the feature. Different training procedures were employed because this experiment was our first attempt to establish nicotine-specific goal tracking to a discrete CS. For example, we felt that the training procedures in the Pre-training group (see later) might facilitate acquisition of nicotine-specific goal tracking. The Discrimination group was included to assess the efficacy of a more traditional drug discrimination training regime. The nicotine training dose was chosen based on robust operant performance in previous work (e.g. Chance et al., 1977; Pratt et al., 1983).

\section{Dipper training}

The first two 50-min sessions were dipper training; rats were trained to access $26 \%$ sucrose (w/v) within $4 \mathrm{~s}$, regardless of their location in the chamber. During the first $25 \mathrm{~min}$ of the initial session, the cubicle door remained open and the experimenter delivered sucrose. For the last $25 \mathrm{~min}$ of this session, the cubicle door was closed and the computer controlled sucrose deliveries using a probability function. The probability of sucrose delivery in a $4-s$ interval started at 0.167 , approximately 2.5 sucrose deliveries/min, and gradually decreased to 0.133 (2 sucrose deliveries/per $\mathrm{min}$ ) over the remainder of the session. Dipper training continued on the following day; however, the probability for sucrose delivery began at 0.133 and gradually decreased to 0.05 (approximately 0.75 sucrose deliveries/min).

\section{Pre-training group}

Modulatory training. Nicotine was injected s.c. $5 \mathrm{~min}$ before each placement in the apparatus for the first sixteen 20-min sessions following dipper training 
(Days 1-16). Each presentation of the light CS was immediately followed by $4 \mathrm{~s}$ access to sucrose. On Days 17-56, half of the experimental sessions were preceded by nicotine administration, the remaining sessions were preceded by saline administration. The order in which these two session types (Nicotine or Saline) occurred was quasi-random across each 8-day cycle, with the constraint that the same type of session did not occur on more than two consecutive days. Each type of session was equally represented within the cycles. The CS was followed by $4 s$ access to sucrose during nicotine sessions (Nic:L + ); CS presentations were not followed by any programmed consequence on saline sessions (Sal:L-). A $4 \mathrm{~s}$ empty interval occurred after CS offset in saline sessions, to equate data sampling periods and total duration across the session types.

On Days 1-12 there were eight CS presentations per session; rats were shifted to four trials per session on Days 13-16 due to high dipper entries during the first 12 sessions. The number of CS presentations per session was then increased to six for the remainder of the training phase (Days 17-56). Four Med-State Notation programs were used to deliver the stimuli and record dipper entries. The programs differed in regard to first trial onset (mean $=120 \mathrm{~s}$, range $=90-150 \mathrm{~s}$ ) and the order of inter-trial intervals (ITIs, CS offset to next CS onset). For each program, ITIs were randomly chosen from a distribution (mean $=154 \mathrm{~s}$, range $=94-214 \mathrm{~s}$ ); each interval occurred at least once per session, with the same interval occurring no more than twice in a session. Across an 8-day training cycle each program controlled one nicotine session (sucrose delivered) and one saline session (sucrose not delivered).

Extinction and testing. Six consecutive feature extinction sessions followed the acquisition phase (Days 57-62). The nicotine feature may 'set the occasion' for responding to the light CS. On the other hand, some associative models would predict that the nicotine feature enhances responding to the light CS because of an excitatory association between nicotine and sucrose (e.g. Rescorla and Wagner, 1972). Accordingly, for both groups we attempted to extinguish any potential goal tracking (i.e. conditioned excitation) that was evoked via a nicotinesucrose association. On each of the six extinction sessions, nicotine was injected s.c. $5 \mathrm{~min}$ before placement in the experimental chamber; the light CS and sucrose were not presented (Nic: -) but dipper entries were recorded. Two post-extinction test sessions (Days 63 and 64) followed feature extinction in counterbalanced order across subjects. These sessions lasted $20 \mathrm{~min}$ and consisted of three presentations of the light CS but not sucrose. One session occurred $5 \mathrm{~min}$ after nicotine administration; the other test occurred $5 \mathrm{~min}$ after saline administration. An equal number of rats received nicotine and saline as the first solution tested.
Retention and testing. Following the post-extinction test (Day 64), rats were given free access to food for 30 days. On Day 94 each rat was weighed and a new $85 \%$ target weight was calculated. Food was then restricted such that rats reached the new $85 \%$ target weight within the next 5 days (Days 95-99). Days 100 and 101 were retention tests, 43 and 44 days from the end of acquisition training, respectively. The procedures for these tests were similar to the post-extinction tests except nicotine and saline were tested in the opposite order for each rat.

\section{Discrimination group}

Modulatory training. For this group, discrimination training began on Day 1 (see Table 1). All 56 sessions were parametrically identical to those described for the Pretraining group; however, these rats began training with intermixed nicotine and saline sessions.

Extinction, retention, and testing. Feature extinction, postextinction, and retention test sessions were identical to the Pre-training group. For the post-extinction test three rats were tested with nicotine first; the remaining two rats were tested with saline. During the 43-day retention interval, rats were treated similarly to the Pre-training group; testing order of the solutions was reversed for the retention tests.

\section{Procedures: Experiment 2}

\section{Dipper training}

Dipper training included three 50-min sessions conducted on consecutive days. All three sessions were entirely computer controlled; the probability function decreased from 0.167 to 0.05 per $60 \mathrm{~s}$ (approximately 2.5 to 0.75 sucrose deliveries/min) over the three sessions.

\section{Modulatory training}

Training for these rats $(n=7)$ was similar to the Discrimination group of Experiment 1, except the CS duration was reduced from the $30 \mathrm{~s}$ to $15 \mathrm{~s}$ and it was presented eight times in each 20 -min training session. Prior to testing there were 24 total training sessions in three 8-day cycles (Days 1-24).

\section{nAChR antagonist tests}

Initial tests assessed the effects of pre-treatment with hexamethonium (HEX, 2.5 or $5 \mathrm{mg} / \mathrm{kg}$ s.c.), mecamylamine (MEG, 0.5 or $1.0 \mathrm{mg} / \mathrm{kg}$ s.c.), or saline (SAL) on nicotine-modulated goal tracking. The highest dose of mecamylamine is behaviorally effective without nonspecific effects on goal tracking in our laboratory (Besheer et al., 2004). During the test phase, training cycles were shifted to 7 days with six training sessions and one test in each cycle. A performance criterion (see later) was assessed on the last four training sessions of each cycle. Saline, mecamylamine, and hexamethonium pre-treatment tests were intermixed in a randomized order for 
each rat. After completing all test conditions, each rat was randomly assigned a new randomized order. Thus, each antagonist dose and saline was tested twice for each rat; average elevation scores from these two tests were used for analyses. The programs controlling test sessions were identical to those for saline training sessions, except that test sessions ended after $4 \mathrm{~min}$ and included only one presentation of the CS. SAL, MEC, or HEX was administered $15 \mathrm{~min}$ before nicotine $(0.4 \mathrm{mg} / \mathrm{kg}) 20 \mathrm{~min}$ before the start of the test session.

\section{Naloxone tests}

In subsequent tests, we assessed the effects of naloxone (NAL; $0.0,0.5,2,4$ or $6 \mathrm{mg} / \mathrm{kg}$ s.c.) pre-treatment on nicotine-modulated goal tracking. Naloxone tests began one cycle after rats completed mecamylamine and hexamethonium testing. Naloxone was administered $5 \mathrm{~min}$ before nicotine (i.e. $10 \mathrm{~min}$ before the start of the session). All other test parameters were identical to the nAChR antagonist tests.

\section{Temporal delay tests}

Following the naloxone tests, each rat was tested with various temporal delays $(0,5,25,50,100,125$ or $200 \mathrm{~min})$ between nicotine administration and the start of the test session. After s.c. injection of $0.4 \mathrm{mg} / \mathrm{kg}$ nicotine, rats were returned to the home cage for the appropriate delay, or were immediately placed in the apparatus $(0-\mathrm{min}$ condition). The 5-min delay test was identical to the delay used in all other training and testing procedures and was included as a baseline condition. Two of the longer delays (50 and $100 \mathrm{~min}$ ) were originally included to assess the temporal persistence of the nicotine cue. However, intervals of 125 and $200 \mathrm{~min}$ were added to the previously randomized test blocks due to maintained conditioned responding at 50 and $100 \mathrm{~min}$.

\section{Performance criterion}

Each rat in Experiment 2 was required to complete a minimum of three 8 -day cycles before criterion was assessed (i.e. Modulatory Training). To calculate the criterion, mean elevation scores were contrasted across nicotine and saline sessions for the last training cycle and each test cycle. In order to meet the test criterion, average elevation scores from nicotine sessions had to be a value of 3 greater than average elevation scores from saline sessions. A similar contrast was conducted on firsttrial data from the last nicotine and saline session of each cycle. The criterion required a minimum difference score of 1 for this first-trial contrast. The criterion elevation scores were chosen a priori, based on scores from Experiment 1. All rats met the criterion during the last cycle of Modulatory Training, rats not meeting both criteria during the test phase remained in the home cage during the following test session.

\section{Results}

\section{Experiment 1}

The different procedures used for groups Pre-training and Discrimination resulted in differential exposure to nicotine (36 and 28 nicotine injections, respectively), different amounts of sucrose access (264 and 192 deliveries, respectively), and different ratios of reinforced to non-reinforced presentations of the CS $39: 5$ and $1: 1$, respectively). Accordingly, data from each group were analyzed separately.

\section{Pre-training group}

Modulatory training. Data from the acquisition phase are presented in Fig. 1A. The initial one-way repeated measures ANOVA revealed a main effect of Session $[F(15,75)=8.3, P<0.001]$ on the first $16(\mathrm{Nic}: \mathrm{L}+)$ sessions. A two-way repeated-measures ANOVA on elevation scores from subsequent sessions revealed a significant main effect of Drug $[F(1,95)=139.20$, $P<0.001]$ and a significant Drug $\times$ Session interaction $[F(19,95)=2.67, P<0.01]$. The main effect of Session was not significant $[F<1]$. To assess potential statedependent effects of nicotine (see Discussion), we compared first-trial elevation scores from the first nicotine session (Nic:L+) and saline session (Sal:L-) of discrimination training. Responding to the light did not differ on the first trial of these initial discrimination sessions, $t<1$ (see inset graph of Fig. 1A).

Extinction, retention and testing. Dipper entries were recorded across each extinction session. The repeatedmeasures ANOVA revealed that the number of dipper entries did not change across the extinction phase $[F<1]$. The average number of dipper entries for this group was $50.5( \pm 11.0)$ on the first session and 57.3 $( \pm 12.7)$ on the last session. Elevation scores from the post-extinction test are presented in the left panel of Fig. 1B. The repeated-measures ANOVA revealed significant main effects of Drug $[F(1,10)=86.63, P<0.001]$ and Trial $[F(2,10)=8.55, P<0.01]$. The Drug $\times$ Trial interaction was also significant $[F(2,10)=4.41, P<0.05]$, denoting a decrease in elevation scores across trials during the nicotine session. Bonferroni's multiple comparisons test confirmed this conclusion: elevation scores on the nicotine session only differed significantly from the saline session on Trial $1[t=6.25, P<0.001]$. These withinsession extinction effects (left panel of Fig. 1B) suggested that data from the first trial of the retention tests would be the most informative. As such, only the first trial of retention test sessions were included in analyses (right panel of Fig. 1B). Elevation scores were similar across retention test sessions $[t<1]$.

\section{Discrimination group}

Modulatory training. Elevation scores from the acquisition phase are presented in Fig. 2A. The repeatedmeasures ANOVA revealed significant main effects of 
Fig. 1



(B) Tests

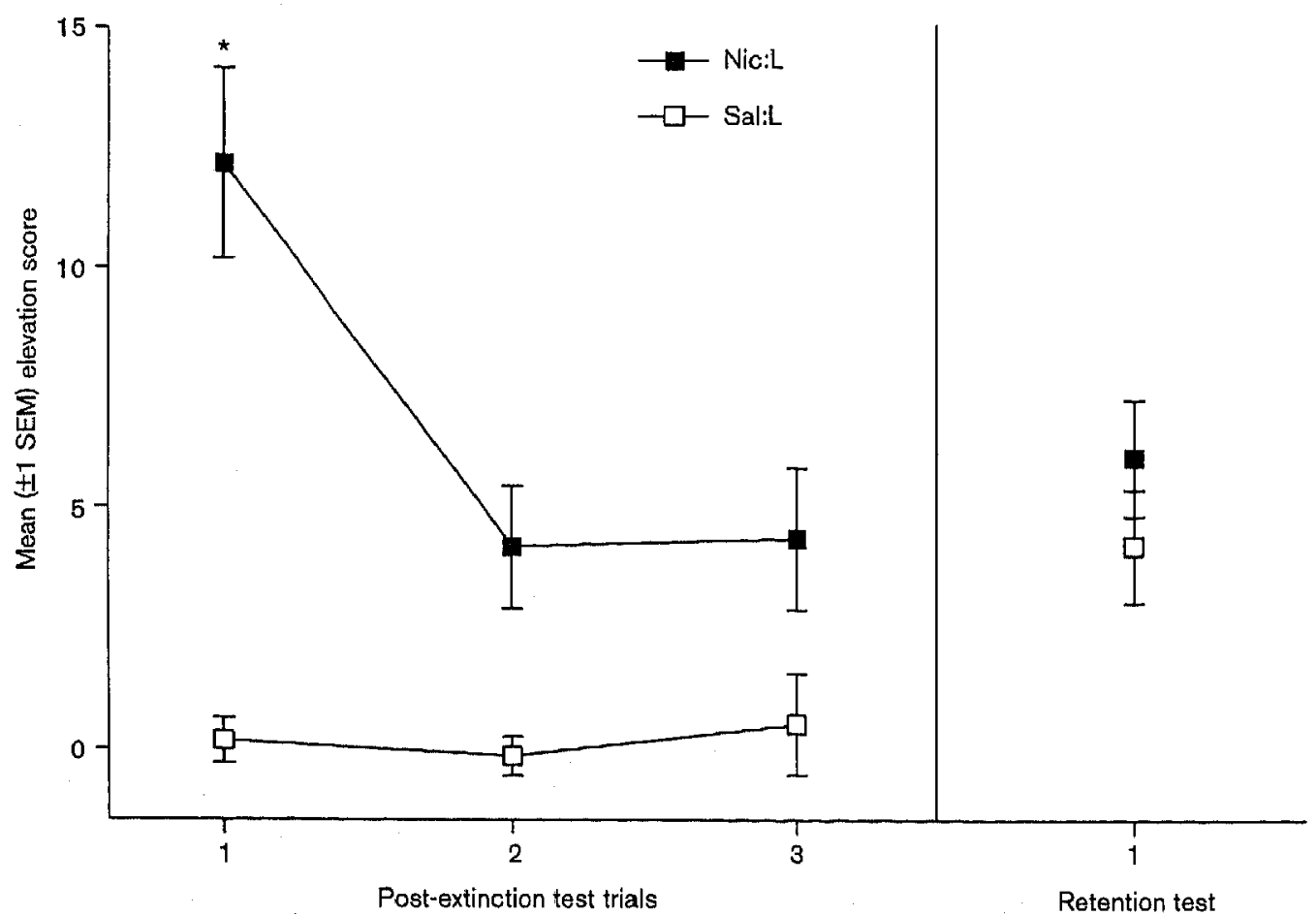

(A) Mean elevation scores ( $\pm 1 \mathrm{SEM}$ ) across training sessions for the Pre-training group of Experiment 1. Rats received a nicotine injection (0.4 mg/ $\mathrm{kg}$ base s.c.) $5 \mathrm{~min}$ prior to 16 training sessions (Days 1-16) in which a light conditional stimulus (CS) was followed immediately by $4 \mathrm{~s}$ access to sucrose unconditional stimulus (US). Across the next $\mathbf{4 0}$ days, nicotine and saline sessions were intermixed; on saline sessions the light CS was presented but was not followed by sucrose. Nicotine and saline sessions occurred on separate days, but are overlaid in order of occurrence to facilitate comparison. The inset graph illustrates first trial elevation scores from the first saline session (session 17) and the 17th nicotine session (session 17). (B) Mean elevation scores from each trial of the post-extinction tests (left), and the first trial of the retention tests (right). $\star$ Indicates that mean elevation score on nicotine session differs significantly from comparable saline session, $P<0.05$. 
Drug $\quad[F(1,108)=9.04, \quad P<0.05]$ and Session $[F(27,108)=7.40, P<0.01]$ and a significant Drug $\times$ Session interaction $[F(27,108)=6.31, P<0.01]$.

Extinction, retention and testing. Dipper entries did not change significantly across extinction sessions $[F<1]$. Average dipper entries were $52.4( \pm 16.1)$ on the first session and $36.8( \pm 5.1)$ on the last session. Elevation scores from the post-extinction test are presented in the left panel of Fig. 2B. The repeated-measures ANOVA revealed significant main effects of Drug $[F(1,8)=38.84$, $P<0.01]$ and of Trial $[F(2,8)=8.85, P<0.01]$. The Drug $\times$ Trial interaction was also significant $[F(2,8)=5.00$, $P<0.05]$, denoting within-session extinction on the nicotine test. Bonferroni's multiple comparisons test revealed that elevation scores on the nicotine session only differed significantly from the saline session on Trial 1 $[t=5.92, P<0.001]$. Elevation scores from the retention test (right panel of Fig. 2B) were significantly higher on the nicotine session than the saline session for this group $[t(4)=2.91, P<0.05]$.

\section{Experiment 2}

\section{Modulatory training}

Data from the acquisition phase are presented in Fig. 3A. The repeated-measures ANOVA revealed significant main effects of Drug $[F(1,66)=72.29, P<0.001]$ and Session $[F(11,66)=6.73, \quad P<0.001]$, as well as a significant Drug $\times$ Session interaction $[F(11,66)=8.96$, $P<0.001]$.

\section{$n A C h R$ antagonist tests}

Elevation scores from the $\mathrm{nAChR}$ antagonist tests are presented in Fig. 3B. The one-way repeated-measures ANOVA was significant $[F(4,34)=7.19, P<0.001]$; follow-up comparisons revealed lower elevation scores in the mecamylamine pre-treatment conditions $(0.5$ and $1 \mathrm{mg} / \mathrm{kg}$ ), relative to saline pre-treatment $\left[P_{S}<0.05\right]$. Hexamethonium did not affect elevation scores $\left[P_{S}>0.05\right]$. Elevation scores from the mecamylamine pre-treatment tests were compared to a 'no-nicotine' baseline for further characterization. This no-nicotine baseline was derived from the first-trial elevation scores of saline training sessions that immediately preceded $1 \mathrm{mg} / \mathrm{kg}$ mecamylamine tests. Pre-treatment with $0.5 \mathrm{mg} /$ $\mathrm{kg}$ mecamylamine yielded significantly higher elevation scores than the no-nicotine control $[P<0.001]$. Elevation scores after $1 \mathrm{mg} / \mathrm{kg}$ mecamylamine pre-treatment were statistically similar to this control $[P>0.05]$.

\section{Naloxone tests}

Elevation scores from the naloxone tests are presented in Fig. 3C. The one-way repeated-measures ANOVA was significant $[F(4,34)=12.04, P<0.001]$, with all doses of naloxone significantly decreasing elevation scores $\left[P_{S}<0.05\right]$. Another no-nicotine control condition was derived from the first-trial elevation scores of saline training sessions that immediately preceded the $6 \mathrm{mg} / \mathrm{kg}$ naloxone tests. Elevation scores from the $0.5 \mathrm{mg} / \mathrm{kg}$ naloxone pre-treatment test were higher than the nonicotine condition $[P<0.05]$. The remaining naloxone pre-treatment doses $(2,4$ and $6 \mathrm{mg} / \mathrm{kg}$ ) yielded similar elevation scores to the no-nicotine control condition $\left[P_{S}>0.05\right]$.

\section{Temporal delay tests}

Data from the temporal effect tests are presented in Fig. 3D. The one-way repeated-measures ANOVA was significant $[F(6,48)=22.06, P<0.001]$; follow-up tests revealed that elevation scores from the $25-$ and $50-\mathrm{min}$ tests were similar to those of the 5 -min condition [PS $>0.05]$. In all other test conditions, elevation scores differed significantly from the 5-min condition $\left[P_{S}<0.01\right]$. Elevation scores from the $100-$ and $125-\mathrm{min}$ tests differed significantly from a no-nicotine control condition $\left[P_{S} \leq 0.001\right]$, derived from first-trial elevation scores of the saline training sessions that preceded 5-min delay tests. The $0-\mathrm{min}$ and $200-\mathrm{min}$ test conditians did not differ from this control $\left[P_{S} \geq 0.05\right]$.

\section{Discussion}

The pharmacological effects of nicotine signaled when a light CS would be followed by sucrose. Accordingly, rats engaged in more food-seeking behaviors (i.e. goal tracking) during the light when nicotine was present than when nicotine was absent. This 'modulatory' control by nicotine developed whether training began with 16 consecutive Nic: $\mathrm{L}+$ sessions or with intermixed Nic:L + and Sal:L- sessions (Experiment 1). Notably, nicotine-specific goal tracking in the Discrimination group seemed to be more variable relative to the Pretraining group. At first glance, this difference might be attributed to the different training parameters. However, in Experiment 2 the training procedures were similar to the Discrimination group but resulted in much less variability. Perhaps more variability in the Discrimination group was due to a longer CS duration $(30 \mathrm{~s}$ in Experiment 1 versus $15 \mathrm{~s}$ in Experiment 2) or to the smaller sample size in this group $(n=5)$; nevertheless, further research will be required to determine the source of this variability. Experiment 2 extended the nicotinespecific goal tracking finding to a shorter CS duration (i.e. $15 \mathrm{~s}$ ) and began to elucidate neuropharmacological mechanisms. To our knowledge, these experiments are the first to investigate the behavioral and neuropharmacological properties of a positive nicotine feature in an appetitive Pavlovian conditioning preparation in rats. Such a preparation could be an important tool for examining the associative and stimulus properties of drugs, given our demonstration of rapid acquisition and stable rates of behavior over a prolonged period of time (e.g. Experiment 2). 
Fig. 2

(A) Acquisition

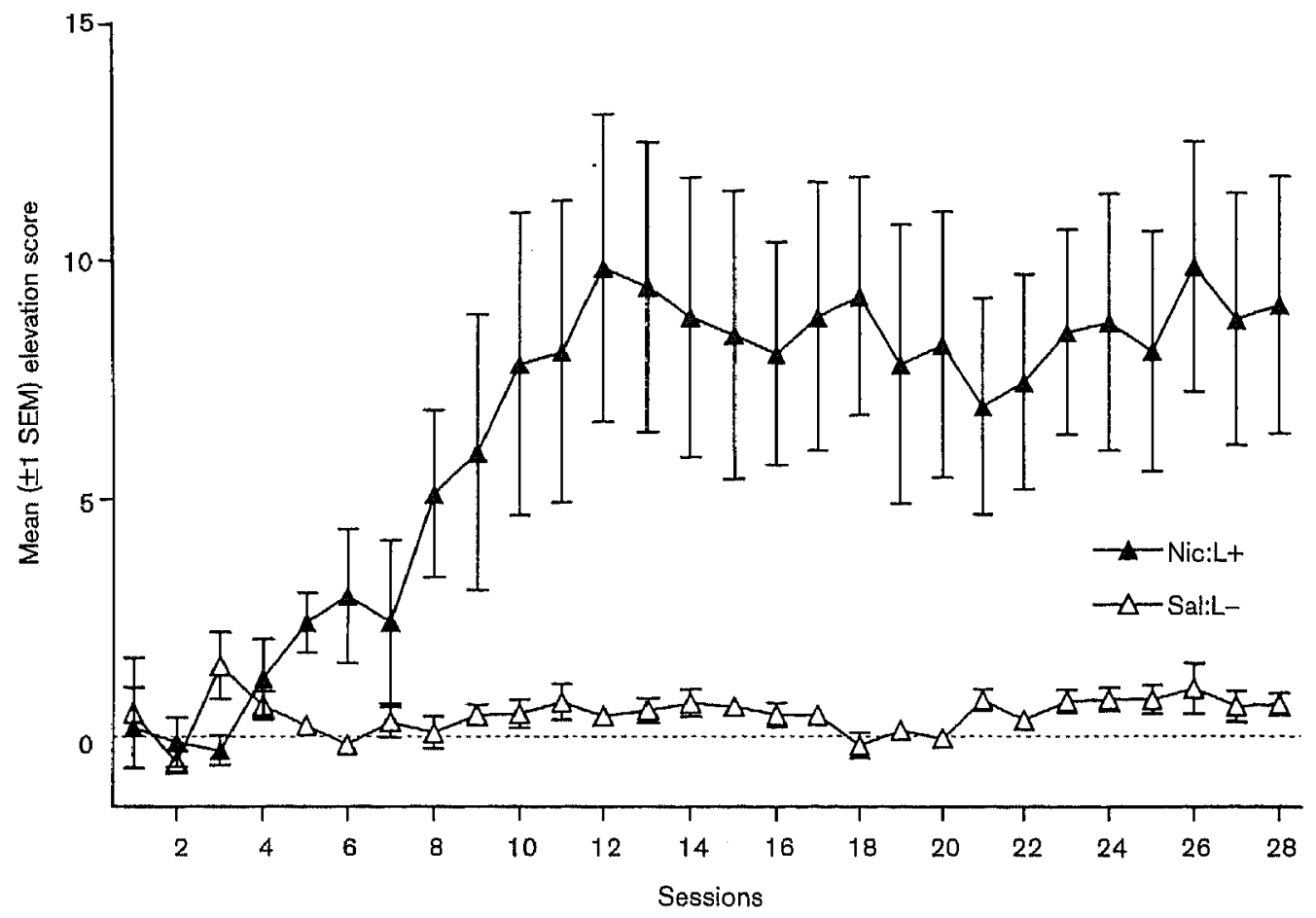

(B) Tests

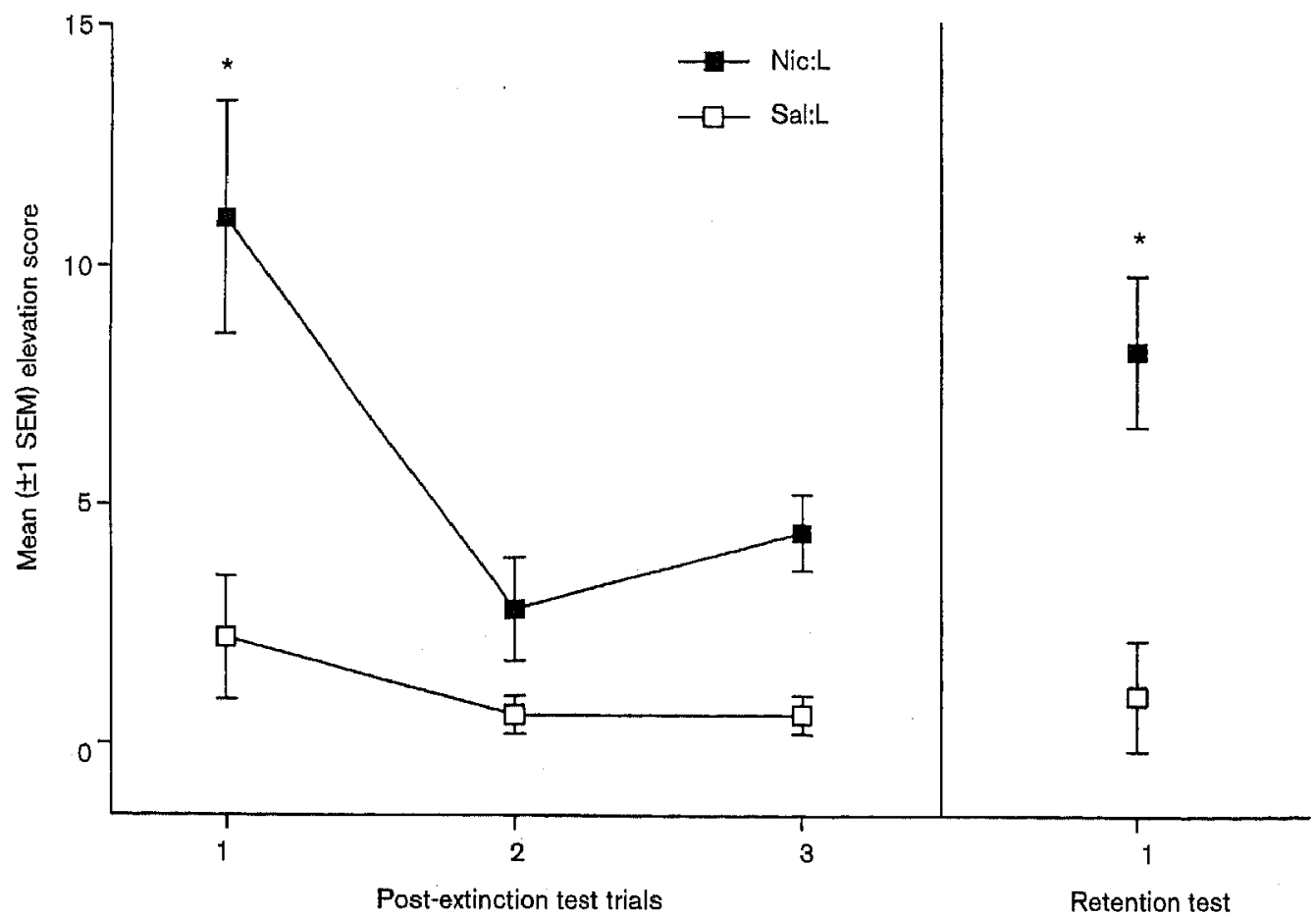

(A) Mean elevation scores ( \pm 1 SEM) across training sessions for the Discrimination group of Experiment 1. For this group, training was identical to that of the Pre-training group (see Fig. 1), except that all nicotine and saline sessions were intermixed. Nicotine and saline sessions occurred on separate days, but are overlaid in order of occurrence to facilitate comparison. (B) Mean elevation scores for each trial of the post-extinction tests (left), and the first trial of each retention test (right). $\star$ Indicates that mean elevation score on nicotine session differs significantly from saline session, $P<0.05$. 


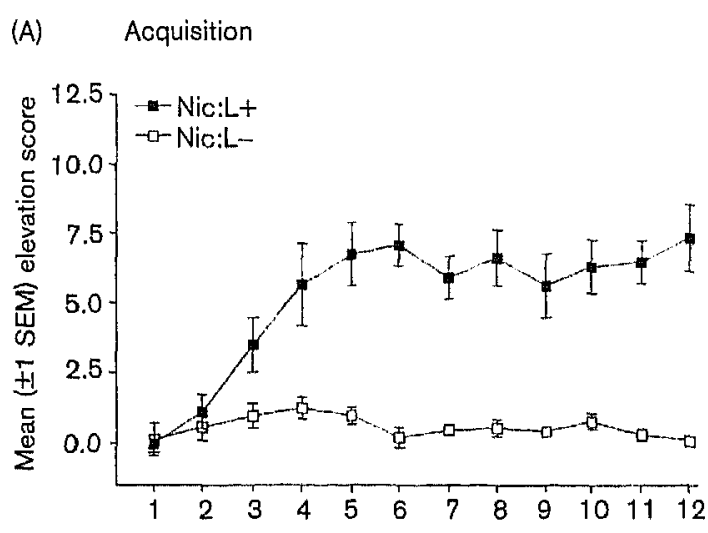

Sessions

(C) Naloxone tests

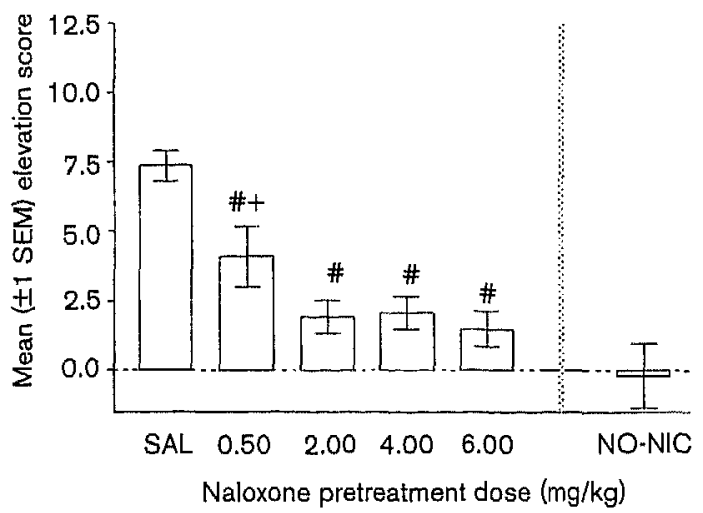

(B) Hexamethonium and mecamylamine tests

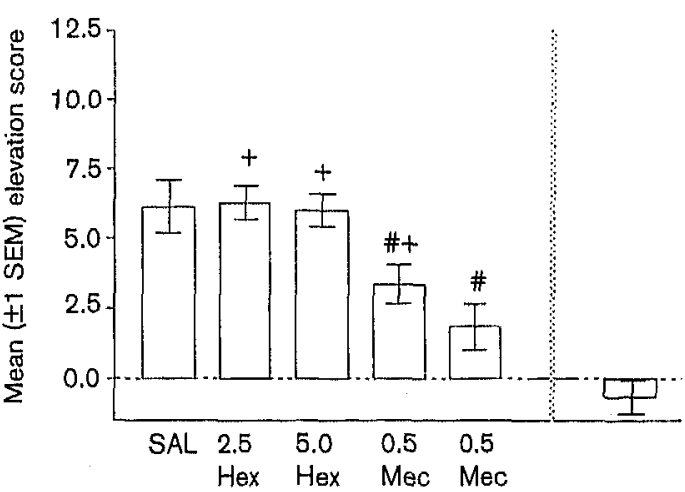

Drug pretreatment dose $(\mathrm{mg} / \mathrm{kg})$ and compound

(D) Delay tests

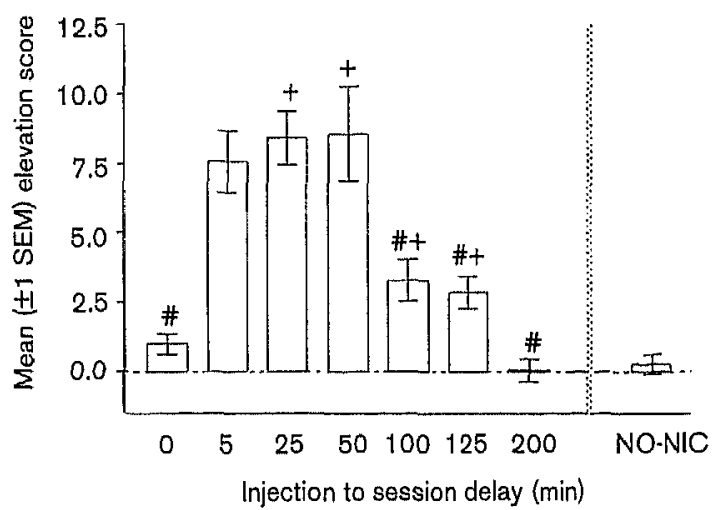

(A) Mean elevation scores ( \pm 1 SEM) across training sessions for rats in Experiment 2. For these rats, training was similar to the Discrimination group from Experiment 1 (see Fig. 2); all rats met the performance criterion by Session 24 and testing began on Session 25. Mean elevation scores on (B) nicotinic acetylcholine receptor ( $n A C h R)$ antagonist pre-treatment tests, (C) naloxone pre-treatment tests, and (D) temporal delay tests are shown. \# Indicates drug pre-treatment or delay condition was significantly different from saline pre-treatment or 5-min delay controls, respectively, $P<0.05 ;+$, indicates drug pre-treatment or delay test condition differs significantly from no-nicotine control condition, $P<0.05$.

In order to fully appreciate the associative and stimulus properties of nicotine in this preparation, we must. examine the means by which nicotine controls goal tracking. Possibly, the pharmacological effects of nicotine provide a stimulus context that modulates a light-sucrose relation (e.g. Bouton and Swartzentruber, 1986). Alternatively, a state-dependent learning account suggests that the association between the light and sucrose is not readily recalled and/or performed outside of the nicotine drug-state (e.g. Cunningham, 1979). Our enthusiasm for this state-dependence account is diminished by some of the results from the Pre-training group in Experiment 1. During the initial nicotine training sessions ( $\mathrm{Nic} \mathrm{L}+$ ), elevation scores for this group increased steadily, suggesting that an association developed between the light CS and the sucrose unconditional stimulus (US). Because of the training protocol, rats in the Pre-training group may have acquired a nicotine-dependent associa- tion. If so, goal tracking to the light CS should be disrupted by removal of the nicotine state. However, goal tracking in the no-drug state did not differ from goal tracking in the nicotine state (see inset graph of Fig. 1A), suggesting that the light-sucrose association was not dependent on the nicotine state.

Another alternative to the modulatory account is based on the perfect correlation between sucrose delivery and the nicotine state. Arguably, higher elevation scores on nicotine sessions could be the result of conditioned excitation evoked by nicotine (e.g. Besheer $e t a l ., 2004$ ) summating with weak excitation evoked by the light CS (i.e. 50\% pairing with sucrose delivery). To test this account, we repeatedly presented the putative conditioned excitor (nicotine) in the absence of the sucrose US. Arguably, this treatment should have reduced the ability of nicotine to evoke a conditional response (i.e. 
extinction; Pavlov, 1927). However, nicotine still controlled goal tracking during the light CS, despite $120 \mathrm{~min}$ of extinction treatment. These data provide preliminary evidence that goal tracking to the light CS was independent of a direct association between nicotine and sucrose. However, we must stress the use of the word 'preliminary'. For example, relative to the training phase (56 sessions) the extinction phase (six sessions) was short. Regardless, this preliminary result is encouraging and we are currently conducting a more complete analysis of nicotine feature extinction.

One reason for the brevity of the extinction phase was our concern about the sensitivity of this task to the passage of time. Since this was the first study of its type in rodents, we had no way of knowing how the passage of time might affect performance. Extensive feature extinction might disrupt modulatory control by attenuating the conditional stimulus properties of nicotine (Pavlov, 1927; Besheer $e t$ $a l ., 2004)$. Alternatively, a long interval between training and testing could affect performance due to decay of associative networks involving nicotine, light CS, and sucrose over time (e.g. forgetting; Bouton, 1994). From this perspective, an important additional test of this preparation was to examine performance after a long retention interval (i.e. 6 weeks). On the retention test, the Discrimination group exhibited more goal tracking to the light during the nicotine session, indicating that the extinction treatment, post-extinction test, and long retention interval did not disrupt the ability of nicotine to occasion the goal tracking CR.

For the Pre-training group, the retention test did not reveal differential goal tracking across nicotine and saline drug states. This failure resulted from high levels of goal tracking during the light on both nicotine and saline test sessions (see right panel of Fig. 1B). This type of retention failure would occur if the first association $(L+)$ was better retained than the subsequent association (L-; e.g. Bouton et al., 1999). Recall that the light CS was initially paired with sucrose on every trial for the Pre-training group, but not for the Discrimination group. Moreover, for the Pre-training group, the light CSsucrose US association was independent of the nicotine state. Accordingly, a retention test might be most sensirive to initial learning, which would be expressed as nicotine-independent goal tracking for the Pre-training group. Although this account fits the data rather well, alternatives such as differential sensitivity to the feature extinction and/or post-extinction tests cannot be eliminated at present.

Many of the behavioral effects of nicotine rely on its action at central nervous system nAChRs. For example, nAChR antagonists that pass the blood-brain barrier (e.g. mecamylamine) attenuate the reinforcing (Corrigall and
Coen, 1989), discriminative stimulus (Stolerman et al, 1984), and avoidance learning (Kumar et al., 1983) effects of nicotine. However, nAChR antagonists that do not pass the blood-brain barrier (e.g. hexamethonium) do nor impact these effects of nicotine (Kumar et al., 1983; Stolerman et al., 1984; Corrigall and Coen, 1989). In Experiment 2, the ability of nicotine to serve as a modulatory cue was dose-dependently attenuated by mecamylamine pre-treatment, but nor by hexamethonium. This pattern is consistent with previous reports that the discriminative stimulus effects (e.g. Stolerman et al., 1984) as well as the conditional stimulus properties (Besheer et al., 2004) of nicotine are mediated by central nAChRs.

Naloxone also attenuated goal tracking occasioned by nicotine in a dose-dependent manner. One reason for this attenuation could be that antagonism of opioid receptors decrements some of the 'downstream' effects of nicotine. For example, stimulation of nicotinic receptors results in the release of endogenous opioids (e.g. Hexum and Russet, 1987); presumably naloxone administration would antagonize the effects of this opioid release. Also, nicotine-induced dopamine release in the nucleus accumbens is attenuated by pre-treatment with naloxone (Tanda and DiChiara, 1998). Blocking these opiaterelated downstream effects of nicotine could attenuate some aspect (e.g. salience) of the nicotine stimulus. Notably, the doses of naloxone that antagonized nicotinespecific goal tracking were much higher than those used to block the effects of morphine and other prototypical mu opioid agonists (e.g. $0.1 \mathrm{mg} / \mathrm{kg}$; Stolerman et al., 2002). Perhaps attenuation of nicotine-specific goal tracking was due to antagonism at both mu and delta opiate receptors (e.g. Takemori and Portoghese, 1984; Tiseo and Yaksh, 1993). Activation of kappa opioid receptors via selective agonists attenuates the effects of nicotine in the central nervous system (e.g. Oka et al., 1998; Maisonneuve and Glick, 1999; Hahn et al., 2000). Therefore, decrement of nicotine-specific goal tracking was probably not the result of naloxone antagonism at kappa receptors. On the other hand, naloxone, which produces a perceptible interoceptive stimulus on its own (e.g. Smurthwaite et al, 1992), may have attenuated goal tracking via generalization decrement (Pavlov, 1927). Pre-treatments with naloxone (especially at higher doses) could make interoceptive nicotine cues dissimilar from the originally trained modulator. This difference between interoceptive cues would result in a loss of goal tracking controlled by nicotine. Distinguishing between these alternatives will require further investigation.

In Experiment 2, changing the time between nicotine injection and the start of the experimental sessions altered the ability of nicotine to control goal tracking to the light CS. The effects of varying the interval between nicotine injections and test sessions are consistent with 
evidence from previous studies on the behavioral and physiological time course of nicotine (Pratt et al., 1983; Ghosheh at al., 1999). For example, a loss of goal tracking on the 0-min test indicates that nicotine had not accumulated to sufficient plasma and brain concentrations to produce the stimulus that occasioned goal tracking (see Pratt $e t$ al., 1983). The attenuation of goal tracking from 100 to 200 min likely reflects significant decreases in the plasma and brain concentrations of nicotine over similar intervals (Ghosheh et al., 1999; Pratt et al., 1983). For example, in an operant nicotine $(0.4 \mathrm{mg} / \mathrm{kg}$ base, s.c.) discrimination using male hooded rats, nicotine-appropriate responding peaked from 2.5 to $10 \mathrm{~min}$ post-injection and reached saline levels by 160 min (Pratt et al., 1983). In the same study, plasma nicotine concentration peaked between 2.5 and 10 min post-injection and declined considerably by $160 \mathrm{~min}$. Using male Sprague-Dawley rats, Ghosheh et al. (1999) determined that brain concentrations of nicotine $(0.8 \mathrm{mg} / \mathrm{kg}$ base, s.c.) peaked at approximately $5 \mathrm{~min}$ postinjection and declined significantly between 60 and $240 \mathrm{~min}$; the brain half-life of nicotine was determined to be approximately $52 \mathrm{~min}$.

These data represent an important extension of previous investigations of drug-modulated Pavlovian conditioning (e.g. Miller et al., 2002; Skinner et al., 1998). Establishing that an appetitive Pavlovian CR can be modulated by nicotine in rodents will facilitate future investigation of the associative and stimulus properties of nicotine. Given that our findings are generally comparable with operant nicotine discriminations (e.g. Pratt et al., 1983; Stolerman et al., 1984), this model might also be well suited for examining the neurobiological substrates of the subjective effects of nicotine. Current research in our laboratory is extending these modulatory properties of nicotine to other drug states, including amphetamine $(1.0 \mathrm{mg} / \mathrm{kg})$ and chlordiazepoxide $(5.0 \mathrm{mg} / \mathrm{kg})$, suggesting that this preparation may be an efficient and informative way to investigate the subjective effects of drugs in general. At minimum, examining the ability of a drug state to control conditioned approach will complement our understanding of its ability to control conditioned avoidance (e.g. Martin et al., 1990) and schedule-controlled behavior (e.g. Pratt et al., 1983).

Further investigations that reveal the associative mechanisms by which drug modulators operate will inform theories of drug abuse, and might also contribute to our understanding of basic Pavlovian conditioning processes. One notable extension of the current studies will be to examine whether nicotine can serve as a 'negative feature'. That is, if sucrose followed the discrete CS in the saline state but not in the nicotine state, would the nicotine cue set the occasion for withholding the goal tracking GR? Such experiments are currently under way in our laboratory, and the answer appears to be affirmative. One question that we have not answered is whether such a training protocol could establish the nicotine cue as a conditioned inhibitor (e.g. Pavlov, 1927). Indeed, the nicotine feature has both a serial and simultaneous relationship with the light CS. Holland and Lamarre (1984) found that negative features presented in simultaneous compound with a CS serve as conditioned inhibirors, whereas negative features presented in serial compound with the CS occasion less conditional responding through some other mechanism. Therefore, the associative and pharmacological mechanisms by which a contextual cue such as nicotine occasions the absence of goal tracking might be considerably different from the manner in which nicotine occasions conditioned approach. Accordingly, research comparing the behavioral and pharmacological processes underlying the ability of nicotine to serve as a positive versus negative feature is needed. Other studies might examine whether drug stimuli can occasion relationships between environmental cues and drug outcomes. More specifically, could the interoceptive effects of alcohol occasion a relationship between the smell of smoke and nicotine delivery? Do drug contexts function similarly to other Pavlovian occasion setters (e.g. Holland, 1999)? That is, would a drug state that occasions conditioned approach with one GS transfer that control to another similarly trained CS (cf. Parker et al., 1994)? Do drug stimuli serve as Pavlovian occasion setters in humans, and are these drug contexts involved in maintaining drug use, relapse, and/or polydrug abuse? These questions about drug stimulus 'contexts' and their role in Pavlovian or stimulus-outcome associations delineate an important and exciting avenue for future research.

\section{Acknowledgements}

We thank Anthony Caggiula and Alan Sved for their thoughtful comments on a previous version of this manuscript and Hannah Siebert and Dawn Metschke for assistance in conducting this research.

\section{References}

Alessi SM, Roll JM, Reilly MP, Johanson CE (2002). Establishment of a diazepam preference in human volunteers following a differential-conditioning history of placebo versus diazepam choice. Exp Clin Psychopharmacol 10:77-B3,

Besheer $J$, Palmatier Ml, Metschke DM, Bevins RA (2004). Nicotine as a signal for the presence or absence of sucrose reward: A Pavlovian drug appetitive conditioning preparation in rats. Psychopharmacology (Berl) 172:108-117.

Bouton ME (1994). Conditioning, remembering, and forgetting. J Exp Psychol Anim Behav Process 20:219-231.

Bouton ME, Swartzentruber D (1986). Analysis of the associative and occasionsetting properties of contexts participating in a Pavlovian discrimination. $J$ Exp Psychol Anim Behav Process 12:333-350.

Bouton ME, Nelson JB, Rosas JM (1999). Stimulus generalization, context change, and forgetting. Psychol Bull 125:171-186.

Brauer LH, Behm FM, Westman EC, Patel P, Rose JE (1999). Naltrexone blockade of nicotine effects in cigarette smokers. Psychopharmacology (Berl) 143:339-346.

Brooks DC, Bouton ME (1994). A retrieval cue for extinction attenuates response recovery (renewal) caused by a return to the conditioning context. $J$ Exp Psychol Anim Behav Process 20:366-379. 
Chance WT, Murfin D, Krynock GM, Rosecrans JA (1977). A description of the nicotine stimulus and tests of its generalization to amphetamine. Psychopharmacology (Berl) 55:19-26.

Corrigall WA, Coen KM (1989). Nicotine maintains robust self-administration in rats on a limited-access schedule. Psychopharmacology (Beri) 99:473-478.

Cunningham $\mathrm{CL}$ (1979). Alcohol as a cue for extinction: State dependency produced by conditioned inhibition. Anim Learn Behav 7:45-52.

Donny EC, Chaudhri N, Caggiula AR, Evans-Martin FF, Booth S, Gharib MA, et al. (2003). Operant responding for a visual reinforcer in rats is enhanced by noncontingent nicotine: implications for nicotine self-administration and reinforcement. Psychopharmacology (Berl) 169:68-76.

Farwell BJ, Ayres JJ (1979). Stimulus-reinforcer and response-reinforcer relations in the control of conditioned appetitive headpoking (goal tracking) in rats. Learn Motiv 10:295-312.

Ghosheh O, Dwoskin LP, Li WK, Crooks PA (1999). Residence times and halflives of nicotine metabolites in rat brain after acute peripheral administration of [2'-(14)C]nicotine. Drug Metab Dispos 27:1448-1455.

Hahn B, Stolerman IP, Shoaib M (2000). Kappa-opioid receptor modulation of nicotine-induced behaviour. Neuropharmacology 39:2848-2855.

Hexum TD, Russet LR (1987). Plasma enkephalin-like peptide response to chronic nicotine infusion in guinea pig. Brain Res 406:370-372.

Holland PC (1999), Intertrial interval effects in Pavlovian serial feature positive discriminations. Anim Learn Behav 27:127-139.

Holland PC, Lamarre J (1984). Transfer of inhibition after serial and simultaneous feature negative discriminative training. Learn Motiv 15:219-243.

Holtzman SG, Locke KW (1988). Neural mechanisms of drug stimuli: experimental approaches. Psychopharmacol Ser 4:138-153.

Kim JA, Siegel S, Patenall VRA (1999). Drug-onset cues as signals: Intraadministration associations and tolerance. J Exp Psychol Anim Behav Process 25:491-504.

Koob GF, LeMoal M (2001). Drug addiction, dysregulation of reward and allostasis. Neuropsychopharmacology 24:97-129.

Kumar R, Pratt JA, Stolerman IP (1983). Characteristics of conditioned taste aversion produced by nicotine in rats. Br J Pharmacol 79:245-253.

Maisonneuve IM, Glick SD (1999). (+/-) Cyclazocine blacks the dopamine response to nicotine. Neuroreport 10:693-696.

Malin DH, Lake JR, Carter VA, Cunningham JS, Wilson OB (1993). Naloxone precipitates nicotine abstinence syndrome in the rat. Psychopharmacology (Berl) 112:339-342.

Malin DH, Lake JR, Upchurch TP, Shenoi M, Rajan N, Schweinle WE (1998). Nicotine abstinence syndrome precipitated by the competitive nicotinic antagonist dihydro-beta-erythroidine. Pharmacol Biochem Behav 60:609-613.

Martin GM, Gans $M$, van der Kooy D (1990). Discriminative properties of morphine that modulate associations between tastes and lithium chloride. $J$ Exp Psychol Anim Behav Process 16:56-68.

Miller MA, Parker BK, Keely JP, Johnson JL, Schall DW (2002). Searching for evidence of transfer between drug facilitators. Learn Motiv 33:197-229.

Oka $K$, Andoh T, Watanabe $I_{1}$ Kamiya $Y$, Ito $H$ (1998). Inhibition of the neuronal nicotinic receptormediated current by kappa opioid receptor agonists in PC12 cells. P/lugers Arch 436:887-893.

Olausson P, Jentsch JD, Taylor JR (2003). Repeated nicotine exposure enhances reward-related learning in the rat. Neuropsychopharmacology 28 $1264-1271$

Olausson $P$, Jentsch JD, Taylor JR (2004a). Nicotine enhances respanding with conditioned reinforcement. Psychopharmacology (Berl) 171:173-178.
Olausson $P_{1}$ Jentsch JD, Taylor JR (2004b). Enhanced responding for conditioned reward produced by intra-accumbens amphetamine is potentiated after nicotine sensitization. Psychopharmacology (Berl) 173:98-104.

Parker BK, Schaal DW, Miller M (1994). Drug discrimination using a Pavlovian conditional discrimination paradigm in pigeons. Pharmacol Biochem Behav 49:955-960.

Pavlov IP (1927). Conditioned Reflexes. London: Oxford University Press

Rescorla RA (1986). Facilitation and excitation. J Exp Psychol Anim Behav Process 12:325-332.

Rescorla RA, Wagner AR (1972). A theory of Pavlovian conditioning: Variations in the effectiveness of reinforcement and nonreinforcement. In: Black $A H$, Prokasy WF (editors): Classical Conditioning II: Current Research and Theory. New York: Appleton-Century-Crofts; pp. 64-99.

Revusky S, Davey V, Zagorski M (1989). Heart rate conditioning with pentobarbital as a conditioned stimulus and amphetamine as an unconditioned stimulus. Behav Neurosci 103:296-307.

Robinson TE, Berridge KC (1993). The neural basis of drug craving: an incentivesensitization theory of addiction. Brain Res Brain Res Rev 18:247-291.

Rose JE, Levin ED (1991). Inter-relationships between conditioned and primary reinforcement in the maintenance of cigarette smoking. Br I Addict 86: 605-609.

Schuster CR, Johanson CE (1988). Relationship between the discriminative stimulus properties and subjective effects of drugs. Psychopharmacol Ser 4:161-175.

Siegel S, Baptista MAS, Kim JA, McDonald RV, Weise-KellyL (2000). Pavlovian psychopharmacology: The associative basis of tolerance. Exp Clin Psychopharm 8:276-293.

Skinner DM, Goddard MJ, Holland PC (1998). What can nontraditional features tell us about conditioning and occasion setting? In: Holland PC, Schmajuk NA (editors): Occasion Seiting: Associative Learning and Cognition in Animals. Washington, DC: American Psychological Association; pp. $113-144$.

Smurthwaite ST, Kautz MA, Geter B, Riley AL (1992). Naloxone as a stimulus in drug discrimination learning: generalization to other opiate antagonists. Pharmacol Biochem Behav 41:43-47.

Stolerman IP, Garcha HS, Pratt JA, Kumar R (1984). Role of training dose in discrimination of nicotine and related compounds by rats. Psychopharmacology (Berl) 84:413-419.

Stolerman IP, Childs $E_{1}$ Hahn B, Morley A (2002). Drug trace discrimination with nicotine and morphine in rats. Behav Pharmacol 13:49-58.

Takemori AE, Portoghese PS (1984). Comparative antagonism by naltrexone and naloxone of $\mathrm{mu}, \mathrm{kappa}$, and delta agonists. Eur I Pharmacol 104: 101-104.

Tanda G, Di Chiara G (1998). A dopamine-mu1 opioid link in the rat ventral tegmentum shared by palatable food (Fonzies) and non-psychostimulant drugs of abuse. Eur J Neurosci 10:1179-1187.

Tiseo PJ, Yaksh TL (1993). Dose-dependent antagonism of spinal opioid receplor agonists by naloxone and naltrindole: additional evidence for delta-opioid receptor subtypes in the rat. Eur J Pharmacol 236:89-96.

Wyvell $\mathrm{CL}$, Berridge $\mathrm{KC}(2000)$. Intra-accumbens amphetamine increases the conditioned incentive salience of sucrose reward: enhancement of reward 'wanting' without enhanced 'liking' or response reinforcement. I Neurosc 20:8122-8130.

Wyvell $\mathrm{CL}$, Berridge $\mathrm{KC}$ (2001). Incentive sensitization by previous amphetamine exposure: increased cue-triggered 'wanting' for sucrose reward. I Neurosci $21: 7831-7840$ 